\title{
Decreased retinal nerve fiber layer thickness in patients with cerebral venous thrombosis
}

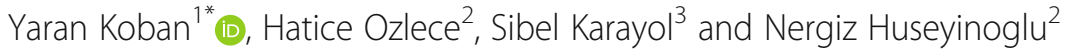

\begin{abstract}
Background: To identify thickness variations in the retinal nerve fiber layer around the optic disc and macula in patients with cerebral vein thrombosis (CVT) without papilledema.

Methods: This study included 28 patients with $\mathrm{CVT}$ diagnosis and appropriate treatment. Detailed ophthalmologic examination found bilateral vision 10/10, vision field test normal and fundus examination found no papilledema images. The patients had macular and optic retinal nerve fiber layer thickness (RNFL) measured with spectral domain-optical coherence tomography (SD-OCT) (Optovue, Fremont, CA). Patients had retinal nerve fiber thickness compared with a control group.

Results: When the effect on the macula and RNFL near the optic nerve disk is investigated, there was significant thinning identified in the macula inferior inner, temporal inner, superior inner and temporal outer quadrants ( $p=0$. $009,0.001,0.026,0.014$, respectively) and in the inferior temporal quadrant of the optic nerve disk $(p=0.020)$ in CVT patients compared to normal individuals.
\end{abstract}

Conclusions: Even after appropriate treatment of CVT patients, axonal loss was identified with OCT. As a result, it may be important to use OCT measurements to monitor CVT treatment.

Keywords: Axonal loss, Cerebral venous thrombosis, Macular thickness, Optical coherence tomography, Retinal nerve fiber layer

\section{Background}

Thrombosis of the cerebral veins and sinuses (CVT) is a rare cerebrovascular disease, that may become significant with missed or late diagnosis. It may be observed in all age groups, more commonly in very young and middleaged individuals and mainly affects women. ${ }^{1}$ Though pregnancy, puerperium, oral contraceptive use, coagulopathies, intracranial infections, cranial tumors, penetrant head trauma, lumbar puncture, malignancy, dehydration, inflammatory bowel disease, connective tissue diseases, Behçet disease, sarcoidosis, nephrotic syndrome, parenteral infusions and a variety of medications have been shown among causes, very detailed investigations have not determined a cause in $20-25 \%$ of cases [1-4].

\footnotetext{
* Correspondence: yarankoban@yahoo.com.au

${ }^{1}$ Faculty of Medicine, Department of Ophthalmology, University of Kafkas,

Merkez, 36100 Kars, Turkey

Full list of author information is available at the end of the article
}

Visual disorders linked to the increase in intracranial pressure like papilledema, diplopia and blurred vision may accompany the tableau $[5,6]$. However, the effect of CVT on the eye in the long term continues to be uncertain. The aim of this study is to assess optic disc retinal nerve fiber and macula thickness variations in patients with CVT without papilledema after appropriate treatment. To the best of our knowledge, the present study is the first to evaluate the posterior ocular segment changes in patients with CVT using optical coherence tomography (OCT) or traditional imaging modalities.

\section{Materials and methods}

This prospective case-control study, which was conducted by the Department of Ophthalmology and Department of Neurology, Kafkas University Medical Faculty Hospital (KUMFH), adhered to the tenets of the 
declaration of Helsinki and was approved by the local ethics committee (80576354-050-99/122; Kafkas University, Human Ethics Comitteee, Kars, Turkey). Our study included all consecutive patients over the age of 18 treated in the KUMFH area for CVT between December 2012 and July 2016. All subjects provided informed consent to participate in the study.

\section{Participants}

The study included 28 patients with CVT diagnosis at Kafkas University Medical Faculty monitored in the Neurology clinic with best improved vision sharpness on Snellen eye chart and 30 healthy volunteers with examination at the ophthalmology clinic. The 28 CVT patients had been admitted to the Kafkas University Medical Faculty Neurology clinic, with definite diagnosis with cranial magnetic resonance imaging (MRI) and MR venography and had common complaints of severe headaches previously unknown or with changed character. The majority of patients had vomiting complaint. Five patients applied to the hospital due to experiencing epileptic seizures. None of the patients had vision complaints. During admission to the neurology ward, patients had consciousness varying from open to stupor, with neurologic deficit present in some patients. Seven patients had radiological diagnosis of thrombosis mainly of the right sinuses (sigmoid, transverse, v. jugularis), with 21 diagnosed with thrombosis of the left sinuses.
Additionally, on radiology some patients had thrombosis of the central sinuses (superior and inferior sagittal, sinus rectus, confluence sinus) (Fig. 1). All patients had anticoagulant treatment while some had antiedema treatment administered. On first neurological examination, no patient had papilledema.

For the study, patients had ophthalmologic examination when general state was stable, when neurological symptoms regressed and after antiedema treatment was stopped. Ophthalmologic examination was completed from 9 to 12 months after the patients applied to the neurology clinic. During ophthalmologic examination, all patients were conscious and had no neurological symptoms.

The selection criteria were normal optic nerve appearance on dilated stereoscopic examination and fundus photography; normal visual field defect at Humphrey perimeter during follow up. None of the patient and control group had thyroid disorders, hypertension, diabetes mellitus, cardiovascular events, Cushing disease, or congenital adrenal hyperplasia. Patients and healthy volunteers who used alcohol or tobacco were excluded from the study. The patients and control subjects who underwent ocular surgery or had ocular trauma or any ocular diseases at the time of OCT measurement were excluded as well. The control group consisted of normal healthy volunteers recruited from among the hospital staff.
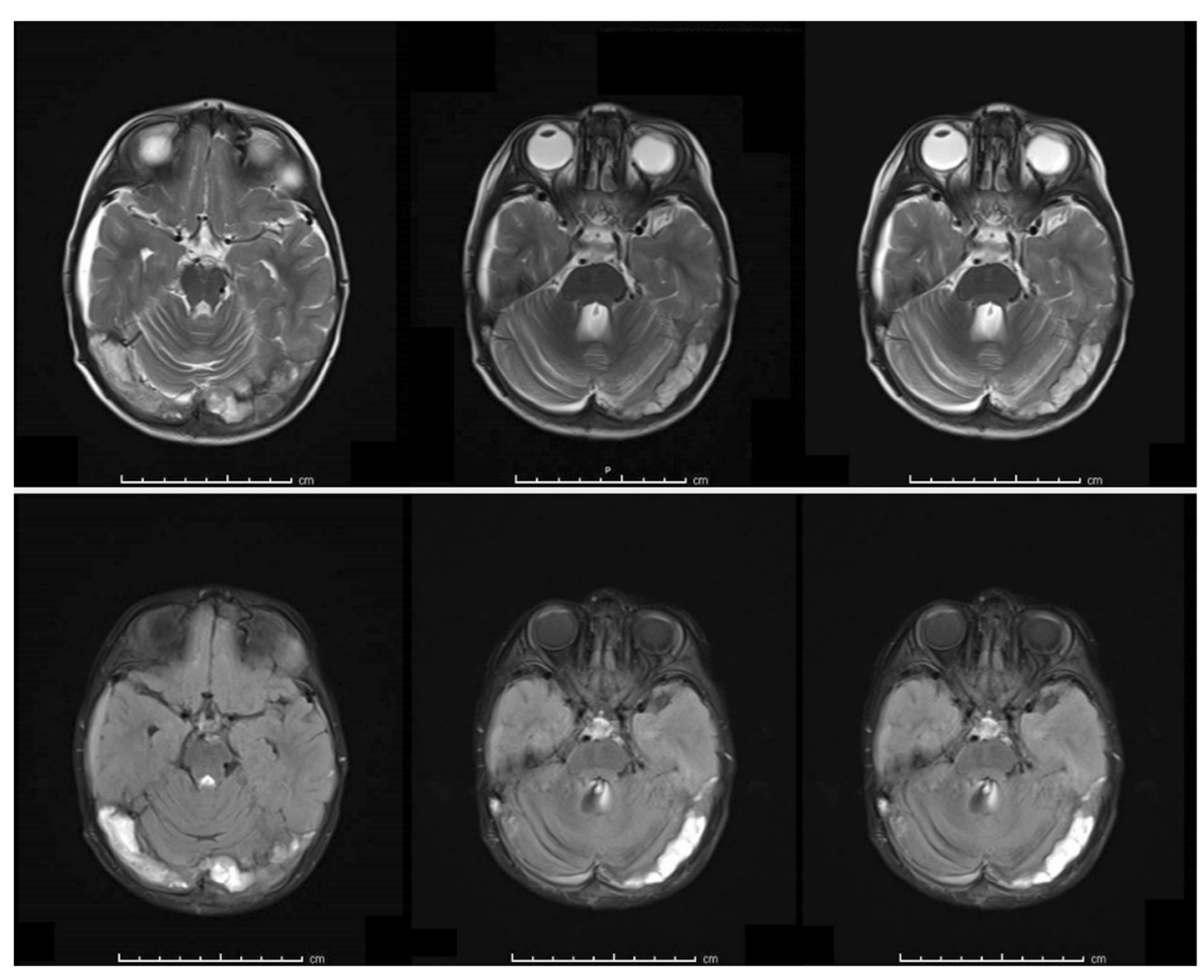

Fig. 1 Increased signal intensity on T1, T2 and FLAIR-weighted series compatible with thrombus in right and left transverse sinuses 


\section{Study protocol}

The diagnosis of CVT had to be confirmed by cerebral MRI combined with cerebral MR venography, following established diagnostic criteria [7]. Each patient underwent a comprehensive ophthalmologic examination. Following this detailed ophthalmologic examination, macular and RNFL thickness were measured using spectral domain OCT with digital software (RTVue-100, Optovue Inc., Fremont, CA, USA). OCT imaging was performed through undilated pupils and with the same intensity of dim room lighting. All images were taken by the same operator. MM6 (12 radial line scans with 1024 A-scans each, within $6 \mathrm{~mm}$ diameter) protocol was used to detect the macular thickness measurements. The scan was divided into the nine Early Treatment Diabetic Retinopathy Study (ETDRS) subfields [8]. The parameters registered in this study were superior outer macular thickness (SOM), inferior outer macular thickness (IOM), temporal outer macular thickness (TOM), nasal outer macular thickness (NOM), superior inner macular thickness (SIM), inferior inner macular thickness (IIM), temporal inner macular thickness (TIM), nasal inner macular thickness (NIM), and central foveal thickness (CFT). The peripapillary RNFL thickness and parameters were calculated by fast RNFL scan, which measures RNFL thickness at $3.45 \mathrm{~mm}$ from the center of the optic disc.

\section{Statistics}

SPSS 11.5 (SPSS Inc., USA) software was used to perform the statistical analyses. The Mann-Whitney $U$ test was used to compare numeric variables between headache and control groups and $P$ values $<0.05$ were considered as statistically significant.

\section{Results}

The mean age of 28 CVT cases with ages from 21 to 36 was $27.72 \pm 5.12$ years, while the mean age of 30 cases in the control group with ages from 18 to 43 was $29.83 \pm$ 9.02 years. Of CVT cases 3 were male (10.7\%) and 25 $(89.3 \%)$ were female. The control group included 5 males (16.7\%) and 25 females (83.3\%). The mean axial length of patients in the CVT group was $23.9 \pm 0.91$, while for healthy volunteers this was $23.9 \pm 0.84$. There was no significant difference in age, gender distribution and axial length between the 2 groups $(p=0.116, \mathrm{p}=$ $0,492, p=0.457$, respectively). Detailed ophthalmologic examination of patients and volunteers in the control group found bilateral vision $20 / 20$, with mean intra ocular pressure in the CVT group $18.0 \pm 0.2 \mathrm{mmHg}$ and in the healthy volunteers $16.9 \pm 2.0(p=0.214)$. Bilateral anterior segment findings were natural. Fundus examination found no papilledema images and visual field tests were normal in both eyes.

\section{Comparison of macular thickness in patients with CVT and healthy volunteers}

We found that all macular thickness parameters were lower in eyes with CVT versus to normal eyes. This thinning in the values was significant for IIM, TIM, SIM, and TOM segments $(p=0.009, p=0.001, p=0.026, p=$ 0.014 , respectively) (Table 1 ). In terms of central, parafoveal and perifoveal macular thickness parameters, there was no statistically significant difference identified between the right and left eyes of CVT patients $(p=0.025)$.

\section{Average Peripapillary RNFL thickness in patients with CVT and healthy volunteers}

We found that inferior temporal (IT) part of the peripapillary RNFL were significantly thinner in the CVT group versus to those in the healthy control group ( $p=$ 0.020). There was no significant difference in superior nasal $(\mathrm{SN})$, nasal upper (NU), nasal lateral (NL), inferior nasal (IN), temporal lateral (TL), temporal upper (TU), and superior temporal (ST) scores between the 2 groups. Table 2 shows sectoral RNFL measurements for patients with CVT and controls. For the right and left eyes of CVT patients, there was no statistically significant difference identified for values for the same peripapillary segments $(p=0.25)$.

\section{Discussion}

This study investigated the macula and optic nerve disc RNFL thicknesses in patients with previous CVT diagnosis and treatment, with no vision complaints or papilledema at time of examination and identified that there was significant thinning in the IIM, TIM, SIM and TOM segments of the macula and the IT sector of the optic nerve disc compared to normal individuals. In light of these results, we conclude that after even effective treatment, axonal loss can be detected by OCT in CVT patients. We consider this retinal axonal loss may have developed as a result of subclinical increased intracranial pressure and chronic papilledema not identified by ophthalmoscopy.

Onset forms and clinical symptoms of CVT is extraordinarily variable, and such variability depends on different factors, such as the location, size, duration, and rapidity of development of thrombus. Obstruction of the intracranial venous drainage system secondary to a thrombus is most commonly observed in the superior sagittal sinuses (70-80\%). Later transverse, sigmoid and at lower rates cavernous sinuses are affected [9]. In one third of cases, more than one sinus is affected [10]. Patients may apply with symptoms linked to increased intracranial pressure (headache, papilledema), focal neurological deficits, seizures, encephalopathy or tableau associated with these situations. Elevated opening pressure is a frequent finding in CVT and is present in > 
Table 1 Results of SD-OCT Macular Thickness (mm) parameters

\begin{tabular}{llll}
\hline Parameters & SVT group Mean SD & Control group Mean SD & P \\
\hline Fovea thickness $(\mathrm{mm})$ & $238.44 \pm 19,89$ & $245.90 \pm 28.57$ & 0.263 \\
Inferior inner macula $(\mathrm{mm})$ & $300.89 \pm 28.93$ & $328.67 \pm 45.37$ & $\mathbf{0 . 0 0 9}$ \\
Temporal inner macula (mm) & $296.33 \pm 13.30$ & $309.17 \pm 12.02$ & $\mathbf{0 . 0 0 1}$ \\
Superior inner macula (mm) & $311.96 \pm 14.90$ & $333.33 \pm 46,29$ & $\mathbf{0 . 0 2 6}$ \\
Nasal inner macula $(\mathrm{mm})$ & $304.37 \pm 57.51$ & $323.17 \pm 12.41$ & $\mathbf{0 . 0 8 6}$ \\
Inferior outer macula $(\mathrm{mm})$ & $267.41 \pm 38.80$ & $284.27 \pm 43.59$ & $\mathbf{0 . 1 3 0}$ \\
Temporal outer macula (mm) & $256.15 \pm 32.22$ & $272.50 \pm 13.50$ & $\mathbf{0 . 0 1 4}$ \\
Superior outer macula $(\mathrm{mm})$ & $276.56 \pm 11.92$ & $290.13 \pm 45.31$ & 0.137 \\
Nasal outer macula $(\mathrm{mm})$ & $290.89 \pm 13.47$ & $298.10 \pm 15.06$ & $\mathbf{0 . 0 6 3}$ \\
\hline
\end{tabular}

SVT Sinus venous thrombous, SD Standart deviation; $p$ :

Significant differences at $p<0.05$ are shown in bold face

$80 \%$ of patients. Headache is the most common symptom of CVT considered to occur linked to intracranial pressure, and is present in approximately $90 \%$ of cases. The most common onset symptom of headache in 70 $75 \%$ and benign intracranial hypertension seen in $20-40 \%$ of patients may be observed as a single symptom [6].

Eye symptoms may accompany the tableau $30.5 \%$ of the time and is observed as a single onset presentation in $21.2 \%$ of all CVT patients. The chief abnormal ocular complaint for $85.9 \%$ is blurring and degeneration of acute vision. It is argued that underlying ocular symptom of CVT are venous obstruction and intracranial hypertension [11]. CVT patients exhibit not only vision loss, metamorphopsia, and diplopia, but also the symptoms of eyeball movement restriction, orbital pain, exophthalmos, chemosis, nystagmus, visual area defect and fundus changes. The symptoms of fundus changes include papilledema, optic atrophy, optic disc hemorr hage, retinal hemorrhage, retinal varices, and macular edema $[11,12]$. The most common symptom in eyes is papilledema [11]. Studies have reported papilledema in $27-68.7 \%$ of CVT cases $[10,11,13]$.

Table 2 Results of SD-OCT RNFL Thickness $(\mathrm{mm})$ parameters

\begin{tabular}{|c|c|c|c|}
\hline RNFL & SVT group Mean SD & Control group Mean SD & $\mathrm{p}$ \\
\hline ST & $153.65 \pm 25.27$ & $149.07 \pm 20.23$ & 0.439 \\
\hline SN & $124.45 \pm 26.91$ & $121.43 \pm 20.92$ & 0.627 \\
\hline TU & $90.19 \pm 17.16$ & $86.83 \pm 13.54$ & 0.399 \\
\hline NU & $83.13 \pm 13.79$ & $83.90 \pm 13.11$ & 0.345 \\
\hline $\mathrm{TL}$ & $81.71 \pm 15.06$ & $82.17 \pm 11.44$ & 0.895 \\
\hline $\mathrm{NL}$ & $76.00 \pm 12.32$ & $73.53 \pm 11.77$ & 0.427 \\
\hline $\mathrm{IT}$ & $146.06 \pm 15.03$ & $156.63 \pm 10.21$ & 0.020 \\
\hline IN & $120.68 \pm 23.66$ & $124.43 \pm 23.22$ & 0.534 \\
\hline
\end{tabular}

The disc swelling in papilledema is primarily due to a rise of intracranial pressure (ICP) which produces axoplasmic flow stasis in the optic nerve head. Visual loss from papilledema can occur even in cases of mild ICP elevation. Elevated ICP causes a number of effects on the visual system, the most severe of which is nerve fiber dysfunction from swelling, progressive loss of retinal ganglion cell somas in the retina and retinal ganglion cell axons within the optic nerve, and optic atrophy [14]. In our study, as in previous studies of CVT patients, papilledema diagnosis was made with ophthalmoscopic examination. None of our patients had papilledema identified, but even after appropriate treatment, compared with normal controls, all patients had significantly reduced macular and RNFL thickness. The cause of this thinning was not assessed with ophthalmoscope at time of application, but may be subclinical papilledema and/or subclinical ICPIS.

Monteiro and Afonso evaluated 52 eyes of patients with resolved chronic papilledema from idiopathic intracranial hypertension. Their results showed significantly reduced macular and RNFL thickness and confirmed the ability of OCT to quantify axonal loss in this condition [15]. They reported that this reduction might be explained by transient ischemia due to intra- and extraaxonal edema resulting in atrophy of the retinal nerve fibers. Their study showed that axonal loss following papilledema can be estimated by OCT-measured macular thickness measurements. In eyes with resolved papilledema demonstrated significantly decreased macular thickness in eight out of nine macular parameters compared to the control group, while OCT-measured RNFL thickness were thinner in six of seven RNFL thickness parameters. In our study, we identified significant thinning in 4 of the 9 macular parameters and 1 of the 8 RNFL parameters assessed in CVT patients. We think the cause of this difference may be still present subclinical ICPIS and/or incomplete resolution of papilledema during OCT measurements.

Coagulation mechanisms are blamed for occurrence of CVT. As a result, anticoagulants are primarily used for 
CVT treatment [16, 17]. In addition to anticoagulants forming the basis of treatment, symptomatic treatment is important. Especially in the acute period antiedema treatment to prevent intracranial hypertension that may occur during clinical progression of the disease may be important. Patients should be monitored carefully for intracranial hypertension and complications; antiedema treatment duration should be set according to clinical assessment, fundus examination and vision field tests [18]. All cases had treatment arranged according to the guidelines and patients were monitored closely for intracranial hypertension. None had papilledema or vision field defects identified. In spite of this, the RNFL and macular layer thinning identified in our study shows that OCT is important for monitoring CVT patients and deciding when to stop antiedema treatment. Because OCT makes it possible to detect mild papilledema that could not be visualized using traditional methods $[19,20]$.

A limitation of the present study is that some patients were on anti-edema treatment. Although, we determined that macular thickness parameters were lower in nearly all eyes with CVT, there might be patients with high intracerebral pressure which might affect the RNFL thickness.

\section{Conclusions}

This study was, to the best of our knowledge, the first evaluation of the macular and peripapillary RNFL thickness changes in CVT patients. In conclusion, when patients with no papilledema on fundus examination, with no visual symptoms or vision field defects are assessed with the more sensitive method of OCT, thinning of macula and RNFL thicknesses was observed. All patients with CVT diagnosis should have detailed eye examination including OCT investigation at time of application and during monitoring. Thus, mild papilledema at time of application and macula thickness changes during check-ups will be identified so treatment and monitoring of patients may be completed with more effective methods and eye and cerebral complications will be prevented. We believe our study will be important for monitoring and treatment planning for patients with CVT accompanied by diseases affecting the optic nerve disc like glaucoma.

\footnotetext{
Abbreviations

CFT: Central foveal thickness; CVT: Cerebral vein thrombosis; IIM: Inferior inner macular thickness; IOM: Inferior outer macular thickness; NIM: Nasal inner macular thickness; NOM: Nasal outer macular thickness; OCT: Optical coherence tomography; RNFL: Retinal nerve fiber layer thickness; SIM: Superior inner macular thickness; SOM: Superior outer macular thickness; TIM: Temporal inner macular thickness; TOM: Temporal outer macular thickness
}

Acknowledgements

No applicable.
Funding

No funding was obtained for this study.

Availability of data and materials

The datasets used and/or analysed during the current study is available from the corresponding author on reasonable request.

\section{Authors' contributions}

YK conducted the literature search and composed the manuscript. YK, HO, SK, and $\mathrm{NH}$ conceived the idea for the manuscript, and critiqued the manuscript. $\mathrm{HO}$, SK and $\mathrm{NH}$ carried out the patient interaction and diagnosis, and final approval of the manuscript. All authors read and approved the final manuscript.

\section{Ethics approval and consent to participate}

The study adhered to the tenets of the Declaration of Helsinki. It was approved by the local ethics committee (Kafkas University, Human Ethics Comitteee, Kars, Turkey), and written informed consent was obtained from all participants before they recruited into the study.

\section{Consent for publication}

Written informed consent was obtained from the patient for publication of this study and any accompanying images.

\section{Competing interests}

The authors declare that they have no competing interests.

\section{Publisher's Note}

Springer Nature remains neutral with regard to jurisdictional claims in published maps and institutional affiliations.

\section{Author details}

${ }^{1}$ Faculty of Medicine, Department of Ophthalmology, University of Kafkas, Merkez, 36100 Kars, Turkey. ${ }^{2}$ Department of Neurology, Acıbadem Kayseri Hospital, Kayseri, Turkey. ${ }^{3}$ Faculty of Medicine, Department of Radiology, University of Harran, Sanliurfa, Turkey.

Received: 23 December 2017 Accepted: 21 January 2019

Published online: 20 February 2019

\section{References}

1. Masuhr F, Mehraein S, Einhaupl K. Cerebral venous and sinus thrombosis. J Neurol. 2004:251(1):11-23.

2. Ferro JM, Canhão P, Stam J, et al. Prognosis of cerebral vein and dural sinus thrombosis: results of the international study on cerebral vein and Dural sinus thrombosis (ISCVT). Stroke. 2004;35(3):664-70.

3. Ferro JM, Canhão $P$, Bousser MG, et al. Cerebral vein and dural sinus thrombosis in elderly patients. Stroke. 2005;36(9):1927-32.

4. Kashkoush Al, Ma H, Agarwal N, et al. Cerebral venous sinus thrombosis in pregnancy and puerperium: a pooled, systematic review. J Clin Neurosci. 2017:39(9):9-15.

5. Agostoni E. Headache in cerebral venous thrombosis. Neurol Sci. 2004; 25(3):206-10

6. Agrawal K, Burger K, Rothrock JF. Cerebral sinus thrombosis. Headache. 2016;56(8):1380-9.

7. Bousser MG, Russell RR. Cerebral venous thrombosis. In: Warlow CP, Van Gijn J, editors. Major problems in neurology. London, UK: WB Saunders; 1997. p. 27-9.

8. Early Treatment Diabetic Retinopathy Study Research Group. ETDRS report no. 7: early treatment diabetic retinopathy study design and baseline patient characteristics. Ophthalmology. 1991;98(5):741-56.

9. Renowden S. Cerebral venous sinus thrombosis. Eur Radiol. 2004;14(2):215-26.

10. Damak M, Crassard I, Wolff $V$, et al. Isolated lateral sinus thrombosis: a series of 62 patients. Stroke. 2009;40(2):476-81.

11. Saposnik G, Barinagarrementeria F, Brown RD Jr, et al. Diagnosis and management of cerebral venous thrombosis: a statement for healthcare professionals from the American Heart Association/American Stroke Association. Stroke. 2011;42(4):1158-92.

12. Wang D, Fang B, Wei $S$. Analysis of clinical features of ocular presentation in cranial venous sinus thrombosis. Eur J Med Res. 2011;16(7):324-7. 
13. Sasidharan PK. Cerebral vein thrombosis misdiagnosed and mismanaged. Thrombosis. 2012;2012:210676.

14. Schirmer CM, Hedges TR 3rd. Mechanisms of visual loss in papilledema. Neurosurg Focus. 2007;23(5):E5

15. Monteiro ML, Afonso CL. Macular thickness measurements with frequency domain-OCT for quantification of axonal loss in chronic papilledema from pseudotumor cerebri syndrome. Eye. 2014;28(4):390-8.

16. Nagaraja D, Sarma GR. Treatment of cerebral sinus/venous thrombosis. Neurol India. 2002;50(2):114-6.

17. Montagud M, Montserrat I, Oliver A. Pregnancy and thrombophilia in women with congenital deficit of antithrombin III, protein C, protein S or plasminogen: analysis of 39 cases. Med Clin. 1993;100(6):201-4.

18. Wall M, Johnson CA, Cello KE, et al. NORDIC idiopathic intracranial hypertension study group. Visual field outcomes for the idiopathic intracranial hypertension treatment trial (IIHTT). Invest Ophthalmol Vis Sci. 2016:57(3):805-12.

19. Vartin CV, Nguyen AM, Balmitgere T, et al. Detection of mild papilloedema using spectral domain optical coherence tomography. Br J Ophthalmol. 2012:96(3):375-9.

20. Menke MN, Dabov $S$, Knecht $P$, et al. Reproducibility of retinal thickness measurements in healthy subjects using spectralis optical coherence tomography. Am J Ophthalmol. 2009:147(3):467-72.

Ready to submit your research? Choose BMC and benefit from:

- fast, convenient online submission

- thorough peer review by experienced researchers in your field

- rapid publication on acceptance

- support for research data, including large and complex data types

- gold Open Access which fosters wider collaboration and increased citations

- maximum visibility for your research: over $100 \mathrm{M}$ website views per year

At BMC, research is always in progress.

Learn more biomedcentral.com/submissions 